\title{
An All Silicon Feedhorn-Coupled Focal Plane for Cosmic Microwave Background Polarimetry
}

\author{
J. Hubmayr · J.W. Appel - J.E. Austermann - J.A. Beall · D. Becker · \\ B.A. Benson - L.E. Bleem - J.E. Carlstrom - C.L. Chang - H.M. Cho · \\ A.T. Crites - T. Essinger-Hileman - A. Fox - E.M. George - N.W. Halverson - \\ N.L. Harrington - J.W. Henning - G.C. Hilton - W.L. Holzapfel - K.D. Irwin * \\ A.T. Lee · D. Li · J. McMahon · J. Mehl · T. Natoli • M.D. Niemack · \\ L.B. Newburgh · J.P. Nibarger · L.P. Parker · B.L. Schmitt · S.T. Staggs · \\ J. Van Lanen · E.J. Wollack · K.W. Yoon
}

Received: 25 July 2011 / Accepted: 14 November 2011 / Published online: 1 December 2011

(1) Springer Science+Business Media, LLC (outside the USA) 2011

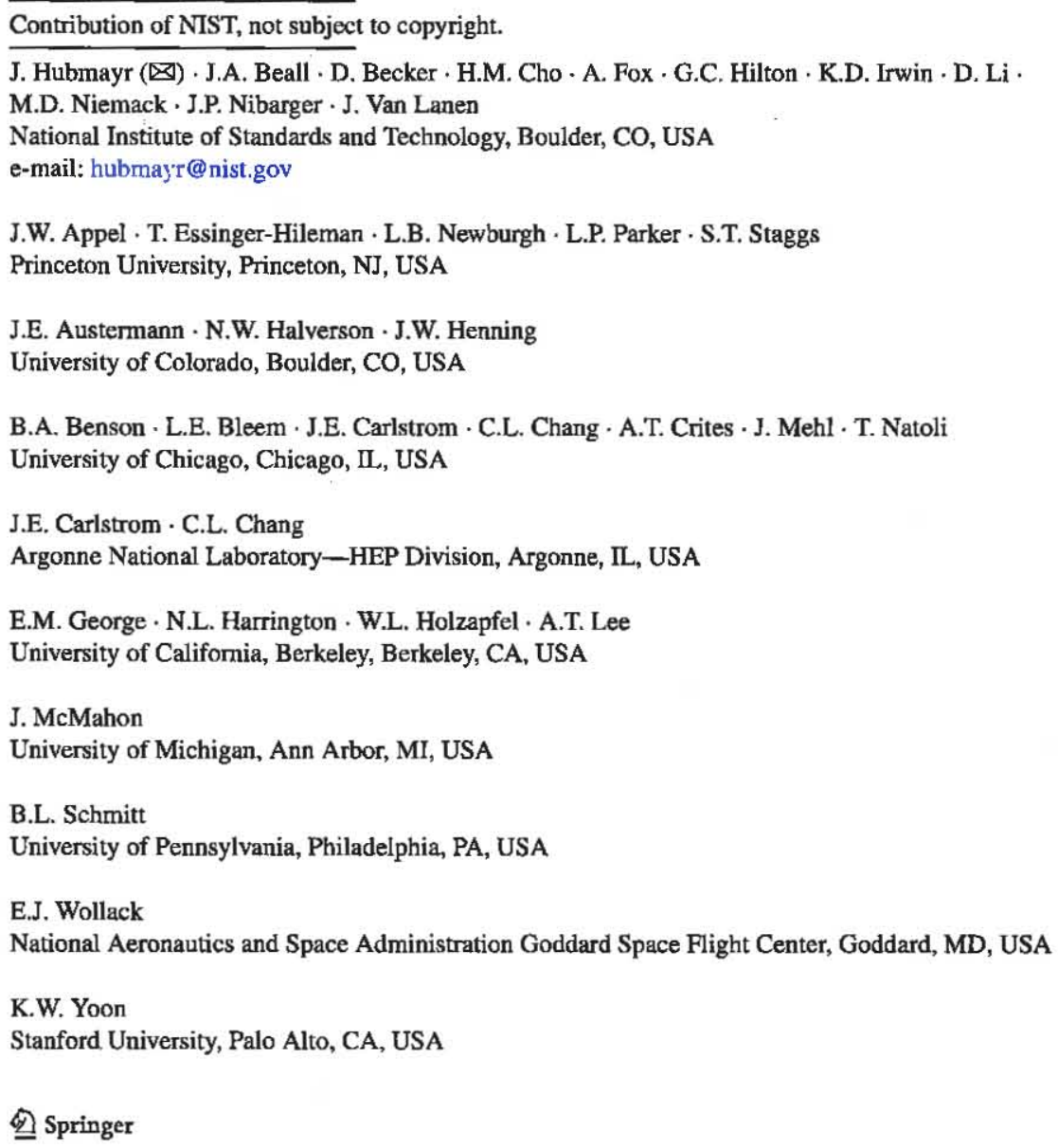


Abstract Upcoming experiments aim to produce high fidelity polarization maps of the cosmic microwave background. To achieve the required sensitivity, we are developing monolithic, feedhorn-coupled transition edge sensor polarimeter arrays operating at $150 \mathrm{GHz}$. We describe this focal plane architecture and the current status of this technology, focusing on single-pixel polarimeters being deployed on the Atacama B-mode Search (ABS) and an 84-pixel demonstration feedhorn array backed by four 10-pixel polarimeter arrays. The feedhorn array exhibits symmetric beams, cross-polar response $<-23 \mathrm{~dB}$ and excellent uniformity across the array. Monolithic polarimeter arrays, including arrays of silicon feedhorns, will be used in the Atacama Cosmology Telescope Polarimeter (ACTPol) and the South Pole Telescope Polarimeter (SPTpol) and have been proposed for upcoming balloon-borne instruments.

Keywords Cosmic microwave background - Polarimeter - Transition edge sensor

\section{Introduction}

Cosmic microwave background (CMB) polarimetry sheds light on the inflationary epoch, neutrino physics and the nature of dark energy. These measurements pose an incredible experimental challenge due to the weak signal strength in the presence of a large background and astrophysical foregrounds. Advances in our understanding of this fundamental physics, from an experimental side, require highly sensitive instruments with strong control over polarization systematic errors.

To meet these technical challenges, we are developing an all silicon, feedhorncoupled focal plane architecture using silicon platelet feedhorns and microfabricated planar orthomode transducers (OMTs) that couple the incident radiation to an array of multiplexed transition edge sensors (TESs) [1]. This monolithic platform is scalable to large arrays, which increases focal plane sensitivity, and minimizes polarization systematics due to the beam properties provided by a single-moded, corrugated feedhorn. The tolerances provided by silicon micromachining yield feedhorns with symmetric beam patterns and excellent array uniformity. Additionally, fabricating from silicon substantially reduces physical and thermal mass, ideal for satellite and balloon-borne applications. Variants of this technology will be used by ABS [2], SPTpol [3] and ACTPol [4] and is proposed for deLITE [5], a balloon-borne CMB polarimeter.

Here we describe this focal plane architecture through a $150 \mathrm{GHz}$ demonstration array composed of a complete 84-pixel monolithic feedhorn array coupled to four, 10-pixel polarimeter arrays. We highlight measurements which characterize the beam properties of the feedhorn array and cryogenic measurements of the polarimeter array.

\section{Technology Overview}

An element in the focal plane is a planar ortho-mode transducer (OMT) coupled to a corrugated feedhorn. Power from the OMT is sensed with transition edge sensors. We implement this concept by fabricating a silicon, corrugated feedhom array and, 


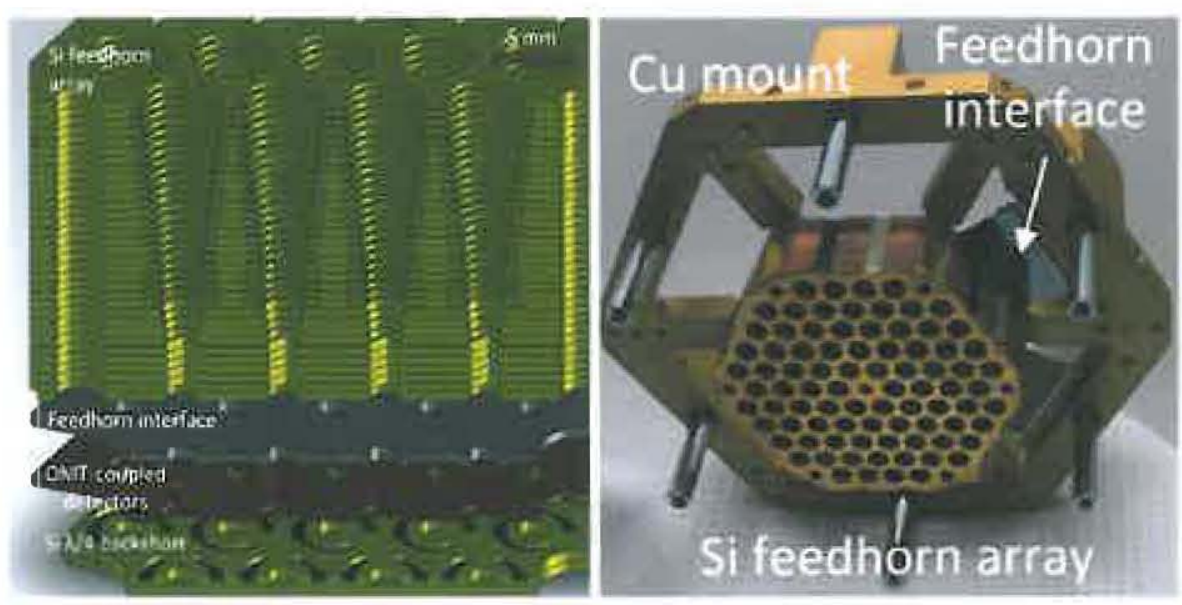

Fig. 1 (Color online) Silicon feedhorn coupled polarimeter array design. Left: This cross-section, expanded model shows the four distinct silicon components that create the silicon feedhorn-coupled focal plane. Right: An image of the 84-pixel hexagonal close-packed silicon corrugated feedhorn array integrated with two polarimeter arrays and mounted to a gold-plated copper ring

separately, a polarimeter array stack composed of a backshort, detector wedge and feedhorn interface as shown in Fig. 1.

Following the successful demonstration of an individual silicon feedhorn [6], we fabricated an 84-pixel silicon platelet corrugated feedhorn array shown in the right panel of Fig. 1. The feedhorn array is composed of a stack of thirty-two $500 \mu \mathrm{m}$ thick silicon wafers with features defined by deep reactive ion etch (DRIE) that are subsequently stacked, aligned and metalized. The feedhorns are designed for singlemoded operation from 125 to $165 \mathrm{GHz}$. Details on fabrication and $300 \mathrm{~K}$ verification of the array are discussed in Nibarger et al. [7].

Coupled to the feedhorn array are 10-pixel polarimeter array stacks. The polarimeter array stack consists of four silicon parts: a backshort cap, backshort, detector wedge and a feedhorn interface as shown in the left panel of Fig. 2. Stacking and aligning these parts creates the OMT in circular waveguide (CWG) of dimensions shown in the right panel of Fig. 2.

Six, 10-pixel hexagonal close-packed detector wedges are fabricated from a $75 \mathrm{~mm}$ diameter, $275 \mu \mathrm{m}$ thick silicon wafer. The $1.6 \mathrm{~mm}$ diameter planar OMTs lie on $0.5 \mu \mathrm{m}$ thick silicon nitride membranes. The center-to-center OMT spacing is $5.26 \mathrm{~mm}$. The backshort cavity is formed by gluing the cap and backshort together. In addition to waveguide, the backshort contains stray light absorbing cavities and $25 \mu \mathrm{m}$ tall radiation blocking structures discussed below. The feedhorn interface contains $250 \mu \mathrm{m}$ tall boss features that are re-entrant with the detector OMT etch to extend the waveguide to the OMT. The resulting structure creates a $25 \mu \mathrm{m}$ gap in the CWG above and below the planar OMT, which is choked to prevent loss at these gaps [8]. The detector wedge outline as well as the features in the support silicon parts are defined by DRIE. The backshort, backshort cap and feedhorn interface are electroplated with $6 \mu \mathrm{m}$ thick gold to ensure good waveguide conductance. Simula- 

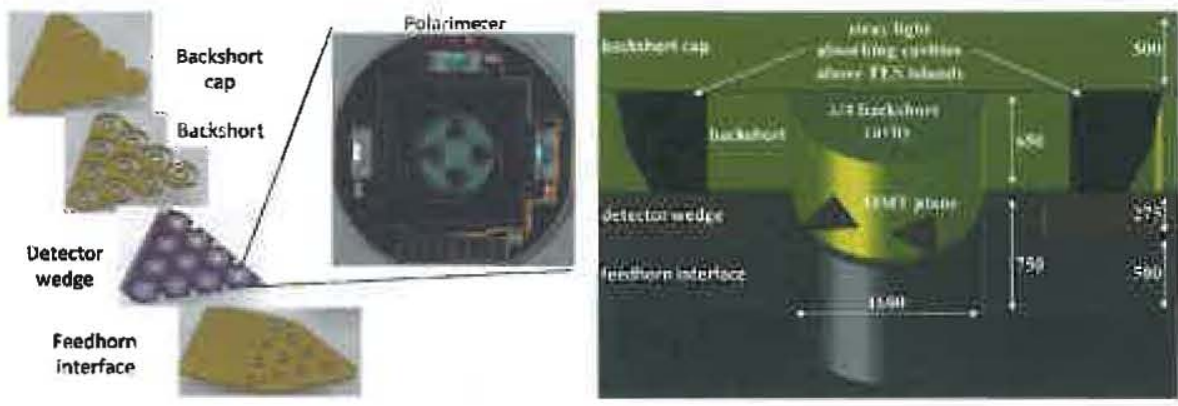

Fig. 2 (Color online) Left: Individual components of a 10-pixel polarimeter array stack. Right: A crosssection, angled model of the OMT in CWG cavity created by the polarimeter array stack. Dimensions are in microns

tions show the required alignment between components to be $25 \mu \mathrm{m}$ [8]. Dowel pin registration restricts misalignment to $6 \mu \mathrm{m}$.

The polarimeter design builds on the previous generation of $150 \mathrm{GHz}$ individual pixels [1]. Single-pixel devices show noise consistent with thermal fluctuation noise [9] and optical band-pass measurements that match simulations [10]. However, improvements can be made on the $57 \pm 9 \%$ band average optical efficiency [11] and the broad spectrum of absorbed power above the pass-band [10].

The 10-pixel polarimeter arrays include several different detector designs in order to probe optical efficiency. One detector design has differential lengths of microstrip in X-pol versus Y-pol channels to explore dielectric loss in silicon oxide $\left(\mathrm{SiO}_{x}\right)$. Another includes an optimized co-planar waveguide for which we expect a $2 \%$ absolute efficiency increase due to improved OMT impedance matching. A third design includes additional gold to niobium microstrip transitions to probe reflections from this interface.

Previous measurements show that out-of-band power is absorbed directly onto the TES island, bypassing the filter. We address this out-of-band leak at the array level by fabricating radiation blocking structures on the backshort that contact the detector wafer around the OMT perimeter. This structure prevents stray radiation from reaching the TES island. Additionally, Eccosorb CR- $117^{1}$ filled cavities below the TESs reduced out-of-band coupling by a factor of two in single-pixel testing. These infrared absorbing cavities are also implemented in the 10-pixel arrays by way of etched cavities in the backshort wafer.

The TES island design varies by experiment to optimize sensitivity and match the multiplexing readout technique. ABS uses Mo:Cu bilayer TESs with resistance suitable for time domain multiplexing (TDM) [12]. SPTpol uses $\mathrm{MHz}$ frequency domain multiplexing (FDM) [13] that requires $\sim 1 \Omega$ sensors, which is achieved by use of Al-Mn alloy TESs [14, 15]. ACTPol also uses Mo:Cu bilayer TESs suitable for TDM; however with transition temperatures, $T_{c}$, near $150 \mathrm{mK}$, a factor of three lower

\footnotetext{
${ }^{1}$ Emerson and Cuming Microwave Products, http://wwu:eccosorb.com. Such identification does not imply recommendation or endorsement by the National Institute of Standards and Technology, nor does it imply that the materials or equipment identified are necessarily the best available for the purpose.
} 
Fig. 3 (Color online) H-plane, E-plane and cross-polar response of the central horn of the silicon corrugated feedhorn array at $150 \mathrm{GHz}$ from [7]. The solid lines are simulations. The cross-polar response deviates from simulation due to mis-alignment in the experimental setup

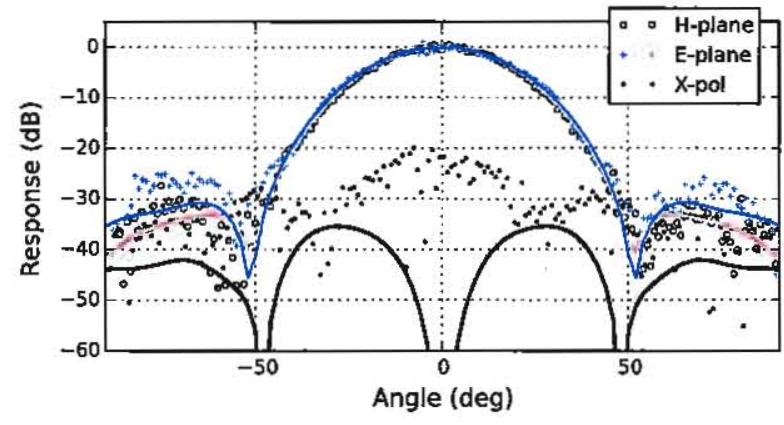

than both ABS and SPTpol. In addition, we have developed low $T_{c}$, low saturation power devices suitable for balloon-borne and satellite applications [16]. The 10-pixel demonstration polarimeter arrays have Mo:Cu TESs with $T_{c} \sim 500 \mathrm{mK}$ and saturation powers $\sim 30$ times larger than the design value for ABS, SPTpol and ACTPol. The large saturation power was chosen to accommodate laboratory optical measurements.

\section{Results and On-going Work}

Measurements of the feedhorn array at $300 \mathrm{~K}$ show $>25 \%$ fractional bandwidth, symmetric beams with $<-23 \mathrm{~dB}$ cross polar response, return loss $<-20 \mathrm{~dB}$ and insertion loss $<-0.4 \mathrm{~dB}$, which we expect to be negligible at sub-Kelvin temperatures [7]. Figure 3 shows that the measured beam maps of the central horn are in excellent agreement with simulation. The measured cross-polar response deviation from simulation is due to alignment accuracy of the experimental setup. The array has been thermally cycled to cryogenic temperatures multiple times with no indication of performance degradation. We are currently expanding the silicon platelet array fabrication techniques to produce ring-loaded structures in the feedhorn throat that provide octave bandwidth for multi-chroic applications. Ring loaded structures have been fabricated as proof of concept, and a $90 / 150 \mathrm{GHz}$ multi-chroic feedhorn design exists.

Optical efficiency measurements at $150 \mathrm{GHz}$ were made by use of an internal cold load [11] on devices with 0,5 and $10 \mathrm{~mm}$ of extra microstrip length from the OMT to the TES island. The measured efficiency is consistent with $\mathrm{SiO}_{x}$ dielectric loss in the microstrip with $\tan \delta=0.01$. Subsequently, we have developed a lower loss $\mathrm{SiO}_{x}$ recipe based on measurements of $\sim 6 \mathrm{GHz}$ microstrip resonators that are quick to fabricate and measure. Through this development process, we expect to improve the optical efficiency to $70 \%$ for pixels with filters and $80 \%$ for filterless polarimeters in which free-space filters must be used to define the pass-band.

The detector properties of four 10-pixel polarimeter arrays have been characterized. Figure 4 shows the wafer-scale uniformity of $T_{c}$ and saturation power, $P_{s a t}$. The saturation power is determined at $80 \%$ of the normal resistance of the TES with a $300 \mathrm{mK}$ bath temperature. We find $T_{c}=495 \pm 7 \mathrm{mK}$ for 87 devices and $P_{\text {sat }}=267 \pm 29 \mathrm{pW}$ for 51 devices. 

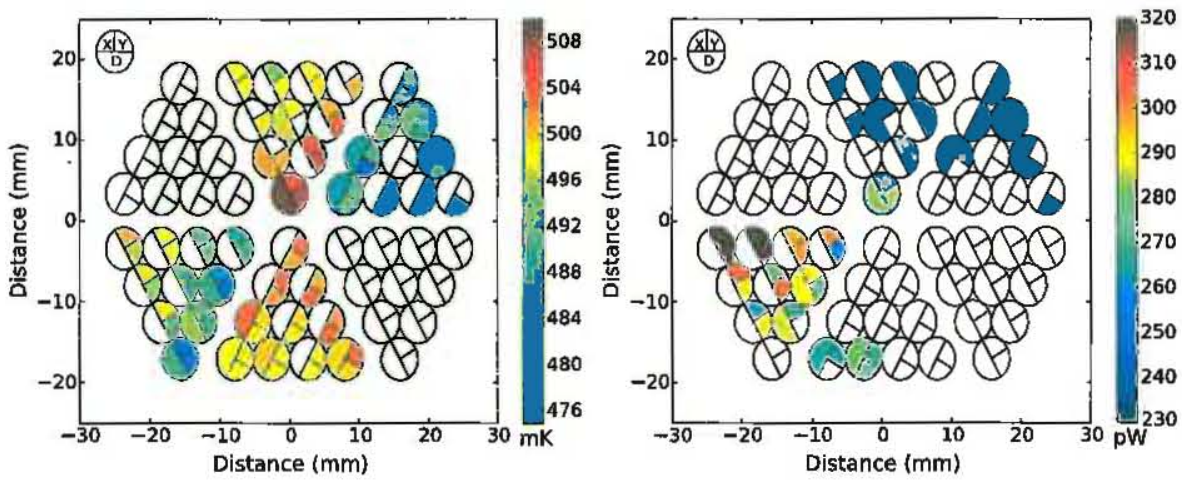

Fig. 4 (Color online) Wafer-scale transition temperature (left) and saturation power (right) uniformity of 10-pixel arrays. The key in the upper left of the plots divides a polarimeter into X-pol, Y-pol and dark TES islands. Colorless sections correspond to unmeasured TESs, not dead pixels

\section{Conclusions}

The first monolithic-silicon corrugated feedhorn array has been fabricated and shows performance that matches simulations. The 10-pixel polarimeter arrays show good uniformity in $T_{c}$ and $P_{s a t}$. We plan to measure beam response functions and array level optical efficiency of the polarimeter array stacks coupled to the feedhorn array using a cryogenic optical dewar in the near future.

The technology will be field tested with ABS, SPTpol and ACTPol, in which both TDM and FDM readout techniques are used. TES islands of low saturation power have been demonstrated and can be integrated with the existing polarimeter design for future balloon-borne or satellite applications. The fabrication techniques are applicable to multi-chroic polarimeters, for which designs of both polarimeters and feedhorns exist.

Acknowledgements Work at NIST is supported by the NIST Innovations in Measurement Science program. The University of Chicago is supported by grants from the NSF (awards ANT-0638937 and PHY0114422), the Kavli Foundation, and the Gordon and Betty Moore Foundation. B.L. Schmidt acknowledges support from NSF GRFP and NASA NSTRF fellowships.

\section{References}

1. K.W. Yoon et al., AIP Conf. Ser. 1185, 515 (2009)

2. T. Essinger-Hileman et al., AIP Conf. Ser. 1185, 494 (2009)

3. J. McMahon et al., AIP Conf. Ser. 1185, 511 (2009)

4. M.D. Niemack et al., SPIE Conf. Ser. 7741, 77411S (2010)

5. S.T. Staggs et al., J. Low Temp. Phys. (2011, this issue)

6. J.W. Britton et al., SPIE Conf. Ser. 7741, 77410T (2010)

7. J.P. Nibarger et al., J. Low Temp. Phys. (2011, this issue). doi:10.1007/s10909-011-0428-z

8. J. McMahon et al., AIP Conf. Ser. 1185, 490 (2009)

9. J.E. Austermann et al., AIP Conf. Ser. 1185, 498, (2009)

10. L.E. Bleem et al., AIP Conf. Ser. 1185, 479 (2009)

11. J.W. Henning et al., SPIE Conf. Ser. 7741, 774122 (2010)

12. W. Doriese et al., Appl. Phys. Lett. 85, 4762 (2004) 
13. T.M. Lanting et al., Appl. Phys. Lett. 86(11), 112511 (2004)

14. D.R. Schmidt et al., IEEE Trans. Appl. Supercond. 21, 196-198 (2011)

15. J. Hubmayr et al., IEEE Trans. Appl. Supercond. 21, 203-206 (2011)

16. M.D. Niemack et al., J. Low. Temp. Phys. (2011, this issue) 